\title{
Periodic subsurface structures in GaAs formed by spatially modulated nanosecond pulse laser irradiation
}

\author{
D.S. Moscal ${ }^{1}$, L.L. Fedorenko², M.M. Yusupov², M.M. Golodenko ${ }^{3}$ \\ ${ }^{1}$ Slovyansk State Pedagogical University, 19, General Batyuk str., 84116 Slovyansk, Ukraine \\ Phone: +380 (06266) 5-06-84; fax:+380 (06262) 9-19-50; e-mail: dsmosk@mail.ru \\ ${ }^{2} V$. Lashkaryov Institute of Semiconductor Physics, NAS of Ukraine \\ 45, prospect Nauky, 03028 Kyiv, Ukraine \\ Phone: +380 (44) 525-64-77; fax:+380 (44) 525-43-82; e-mail:lfedor@isp.kiev.ua,ny@isp.kiev.ua \\ ${ }^{3}$ Donbas National Academy of Civil Engineering and Architecture \\ 2, Derzhavin str., Makiivka, 86123 Donbas region, Ukraine \\ Phone: +380 (0622) 90-29-38, +380 (0623) 22-24-67 \\ Fax: (06232) 4-46-82; e-mail: mailbox@dgasa.dn.ua
}

\begin{abstract}
We used the method of nets to calculate the thermoelastic stresses on the GaAs surface caused by a non-destructive nanosecond pulse laser irradiation $(\lambda=0.532 \mu \mathrm{m})$ with diffraction spatial intensity modulation from a shield with rectangular cut. The structure of irradiated subsurface layers of samples was studied by the AFM method. A periodic islet structure formed in the process of diffusive redistribution of defects was revealed by the level-by-level chemical etching.
\end{abstract}

Keywords: laser, diffraction, intensity modulation, GaAs, thermoelastic stress, subsurface layer, point defect, islet structure.

Manuscript received 07.06.07; accepted for publication 27.09.07; published online 30.11.07.

\section{Introduction}

Laser technologies of creation of semiconductor structures allow one to set the required energy distribution on irradiated surfaces. It was succeeded to form nanostructures with specific properties on the GaAs surface by different methods of laser radiation intensity modulation [1-5]. For a modification of the thin subsurface layer of a semiconductor, it is necessary to use the fundamental absorption of short laser radiation pulses with a certain energy distribution on the irradiated surface $[5,6]$. The present paper deals with researching the structural changes in subsurface GaAs layers caused by diffraction-modulated laser nanosecond pulses with the quantum energy exceeding the semiconductor band gap. The holographic energy distribution creates locally warmed-up regions on the irradiated crystal surface. This results in the origination of thermoelastic stresses in the sample subsurface layer, which stimulates the generation and the diffusive redistribution of point defects [7].

\section{Experiment}

We studied the influence of the pulse laser radiation passed through a diffraction mask on the semiconductor surface (Fig. 1). A chemically polished surface (100) of GaAs brand AGChT-1-25a-1 was irradiated. A rectangular mask was set at a distance of $10 \mu \mathrm{m}$ above the irradiated surface. The laser radiation wavelength corresponded to the resonance light absorption by the semiconductor surface. The irradiation energy density on the diffraction mask was $160 \mathrm{~mJ} / \mathrm{cm}^{2}$. A short-term metallographic etching in $\mathrm{AB}$ solution was used [8] to investigate the structural changes appeared in the subsurface layer at a depth of $\sim 1 \mu \mathrm{m}$. The chemically treated surface was explored by atom force microscope. A periodic islet structure was exposed as a result (Fig. 2).

\section{Results and discussion}

A computer modeling of thermal fields arising in the GaAs subsurface layer under exposure of the absorbed diffraction-modulated laser radiation was performed to clarify the processes of formation of subsurface structures. We have solved the heat conduction equation [9] $c \rho \frac{\partial T}{\partial t}=\frac{\partial}{\partial z}\left(\kappa \frac{\partial T}{\partial z}\right)+g$, where $c$ is the specific heat capacity, $\rho$ is the matter density, $T$ is the temperature, $t$ is the time, $z$ is the co-ordinate in the direction normal to 


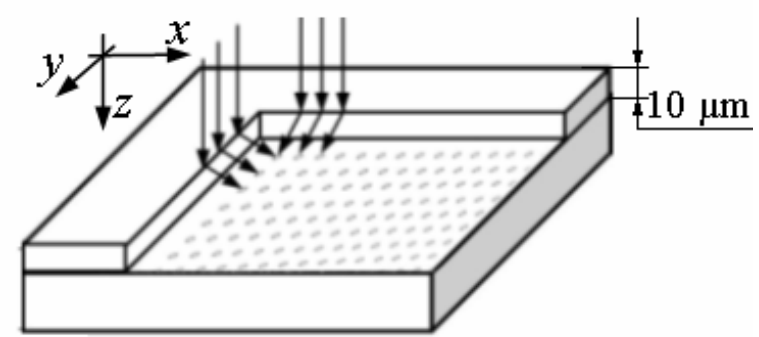

Fig. 1. Scheme of the irradiation of a sample through a diffraction mask with rectangular cut.

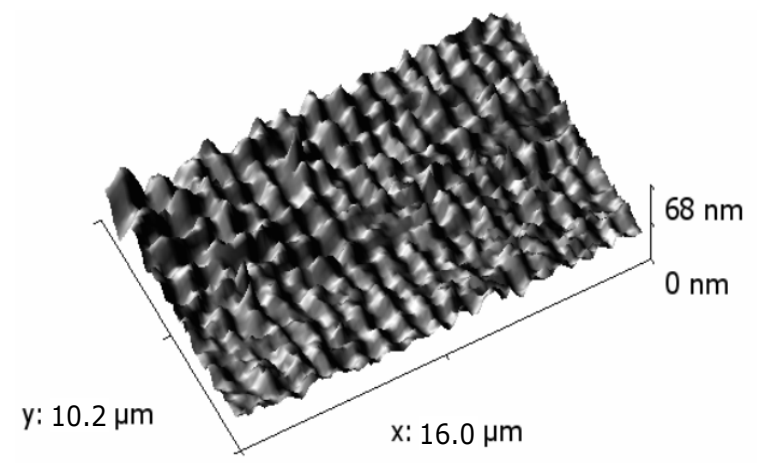

Fig. 2. AFM-image of the GaAs subsurface structure formed after the laser irradiation through a diffraction mask with a subsequent short-term chemical etching.

the surface, $\kappa$ is the heat conductivity, and $g$ is the thermal power released per unit volume. To consider the dynamic processes and changes of the physical properties of the semiconductor during the influence of a laser radiation pulse, the analytical expressions for temperature dependences of parameters (Fig. 3) were used and the heat conduction equation has been solved by the method of nets by the implicit calculation scheme. The diffraction modulation of laser radiation was characterized by the coefficient of intensity distribution $\gamma$. When a laser beam passes through a rectangular cut diffraction mask, the coefficient $\gamma$ is calculated by the formula [10]

$\gamma=\prod_{i=1}^{2} \frac{I_{1}}{I_{0}}=\frac{1}{4} \prod_{i=1}^{2}\left\{\left[\frac{1}{2}+\xi\left(\vartheta_{i}\right)\right]^{2}+\left[\frac{1}{2}+\eta\left(\vartheta_{i}\right)\right]^{2}\right\}$,

where $I_{i}$ is the intensity produced by diffraction on one of the cut sides; and the parameters $\xi\left(\vartheta_{i}\right)$ and $\eta\left(\vartheta_{i}\right)$ are determined in terms of Fresnel integrals. Computations were conducted in accordance with the terms of the experiment. A periodic distribution of light energy density was determined as a result. Its period on the surface of semiconductor is stabilized at a distance $\sim 24 \mu \mathrm{m}$ along the bisector of the diffraction mask rectangular cut (Fig. 4).
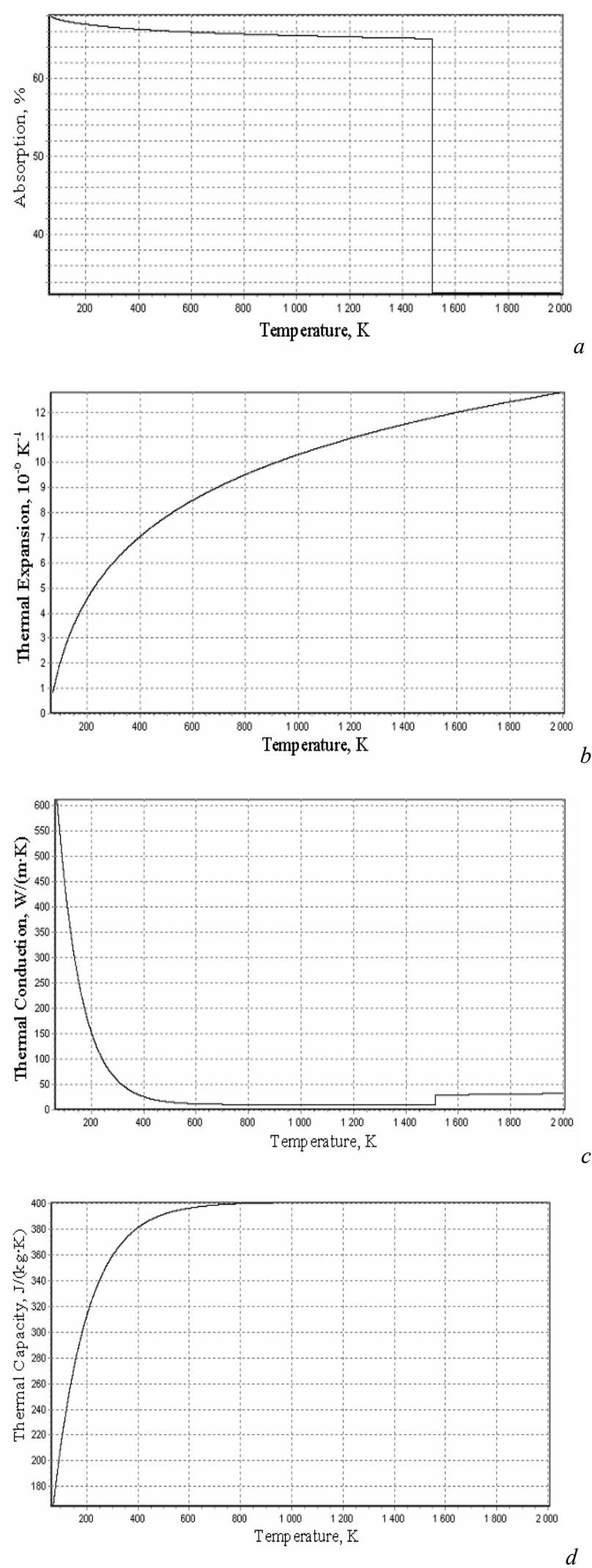

Fig. 3. Temperature dependences: a) light absorption for a wavelength of $0.54 \mu \mathrm{m}$, b) thermal expansion, c) heat conductivity, d) heat capacity. 


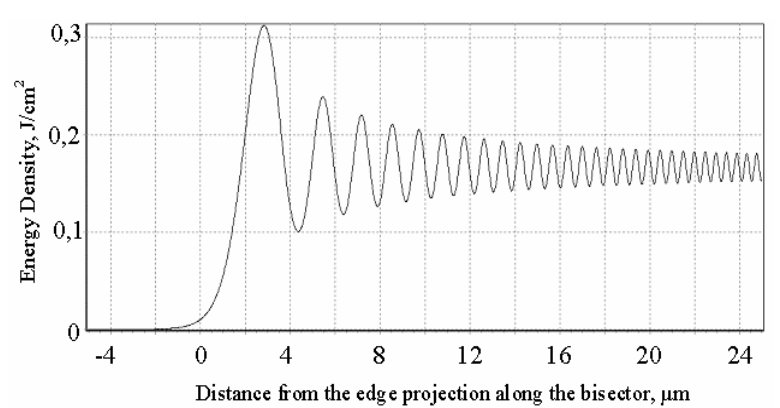

Fig. 4. Falling energy density depending on the distance along the bisector of a diffraction mask with rectangular cut.

While calculating, we have considered also the absorption of the laser light in GaAs vapors. If we take into account only the single ionization of atoms in vapors, then the absorption coefficients of radiation in the vapors for each of the component at the unit of distance can be written down in the form [11]

$$
\begin{aligned}
& \chi_{3}=\frac{e^{6} k T_{\mathrm{V} 3} n_{\mathrm{V} 3}}{3 \sqrt{3} h^{4} c v^{3} 4 \pi \varepsilon_{0}^{3}} \exp \left(-\frac{A_{3}-h v}{k T_{\mathrm{V} 3}}\right) \text { and } \\
& \chi_{5}=\frac{e^{6} k T_{\mathrm{V} 5} n_{\mathrm{V} 5}}{3 \sqrt{3} h^{4} c v^{3} 4 \pi \varepsilon_{0}^{3}} \exp \left(-\frac{A_{5}-h v}{k T_{\mathrm{V} 5}}\right),
\end{aligned}
$$

where $e$ is the elementary charge, $k$ is the Boltzmann constant, $h$ is the Planck constant, $c$ is the light velocity in vacuum, $\varepsilon_{0}$ is the electric constant, $v$ is the laser optical radiation frequency, $A$ is the energy of ionization of an atom, $n_{\mathrm{V} 3}$ and $n_{\mathrm{V} 5}$ are atom concentrations in the semiconductor vapors at the moment of their sublimation from the surface, and $T_{\mathrm{V} 3}$ and $T_{\mathrm{V} 5}$ are the temperatures of vapors of elements $A_{3}$ and $B_{5}$, respectively.

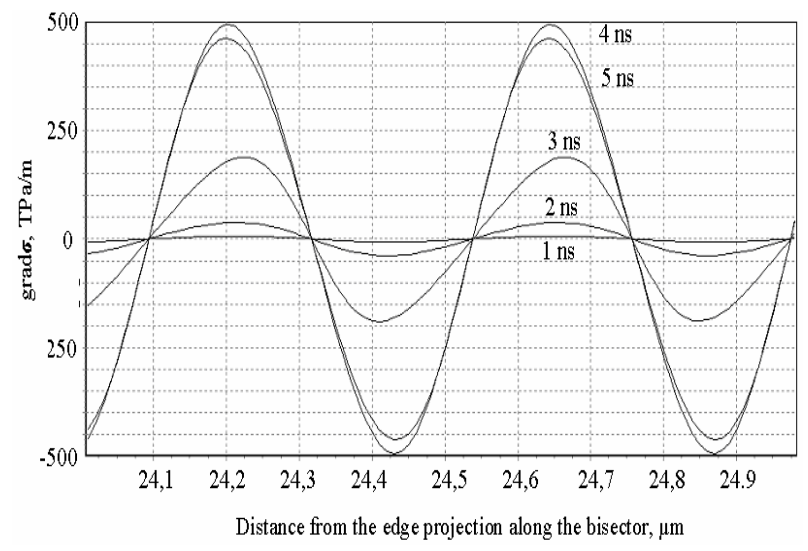

Fig. 5. Distribution of the thermoelastic stress gradient along the bisector of a diffraction mask with rectangular cut.

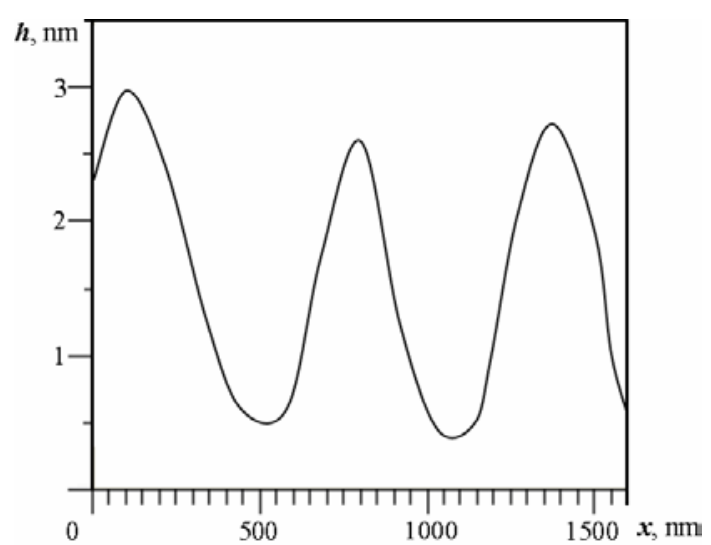

Fig. 6. AFM-profile of GaAs surfaces at a distance of $\sim 24 \mu \mathrm{m}$ from a diffraction mask with rectangular cut.

Only a thin subsurface layer (thickness $\delta \sim \alpha^{-1}$ ) confined on the surface by a laser spot with the diameter $\varnothing \geq 100 \mu \mathrm{m}$ that lies on the massive bedding is heated under the action of pulse laser irradiation. Therefore, we have considered the one-dimensional case. Taking the temperature dependence of the linear thermal expansion coefficient into account, it is possible to calculate a relative deformation of compression [12] $\varepsilon=\int_{T_{0}}^{T} \alpha(T) d T$, where $\alpha(T)$ is the linear thermal expansion coefficient as a function of temperature. The maximum mechanical stress arises in directions [010] and [001] under the irradiation of crystal plane (100). The tension tensor components are calculated as follows [13]: $\sigma_{22}=\sigma_{33}=\left(c_{11}+c_{12}\right) \varepsilon$, where $c_{11}$ and $c_{12}$ are the elasticity tensor components. The distribution of thermoelastic stress gradient is given by the spatial modulation of the irradiation intensity since the periods of the diagrams represented in Figs. 4 and 5 coincide. The heterogeneous stress distribution on the sample surface results in the generation and the diffusive alteration of point defects in the semiconductor [11]. A subsurface structure appears as a result. The arrangement of the experimentally exposed structures coincides with the distribution of thermoelastic stress gradient that is confirmed by the AFM-profiles presented in Fig. 6 . Consequently, the subsurface islet structures appear as a result of the absorption of radiation with diffraction modulated intensity by the semiconductor.

\section{Conclusions}

Under exposure of the nanosecond laser pulse radiation with spatially modulated power density, a periodic islet structure appears in the subsurface layer. The structure period is determined by the diffraction intensity distribution on the irradiated surface. In contrast to other applications of interference effects [14], we used the laser radiation with under-threshold energy density. The 
obtained results can be used for the development of laser technologies for the fabrication of microelectronic devices.

\section{References}

1. P.V. Santos, A.R Zanatta., U. Jahn, A. Trampert, F. Dondeo, I.J. Chambouleyron, Laser interference structuring of $\alpha$-Ge films on GaAs // Appl.Phys. 91 (5), p. 2916-2920 (2002).

2. M.K. Kelly, J. Rogg, C.E. Nebel, M. Stutzmann, Sz. Kátai, High-resolution thermal processing of semiconductors using pulsed-laser interference pattering // Phys. status solidi (a), 166 (2), p. 651657 (1998).

3. S.V. Vintsents, A.V. Zaitseva, V.B. Zaitsev, Genesis of nanoscale defects and damage in GaAs under the multi-pulse quasistatic photodeformations of the micron-sized areas of semiconductor // Fizika Tekhnika Poluprov. 38 (3), p. 257-264 (2004) (in Russian).

4. V.A. Volodin, E.I. Gatskevich, A.V. Dvurechenskiy, M.D. Efremov, G.D. Ivlev, A.I. Nikiforov, D.A. Orehov, A.I. Yakimov, Modification of nanocluster of $\mathrm{Ge}$ in $\mathrm{Si}$ under action of pulse laser radiation // Ibid. 37 (11), p. 1352-1357 (2003).

5. V.D. Andreeva, M.I. Anisimov, N.G. Dgumamuhambetov, A.G. Dmitriev, Structure of the GaAs〈Te $\rangle$ crystals modified by the pulse laser radiation // Ibid. 24 (6), p. 1010-1013 (1990).
6. K. Sadra, Lateral resolution in laser-patterned thermal processing of GaAs // Appl. Surf. Sci. 125 (3-4), p. 325-331 (1998).

7. V.A. Nadtochiy, V.P. Alehin, Microplasticity in Ge single crystals subjected to laser irradiation and deformation of compression // Fizika Khimiya Obrabotki Mater. 4, p. 27-32 (2004) (in Russian).

8. Z.Yu. Gotra, Technology of Microelectronic Devices: Reference Book. Radio i Svyaz, Moscow (1991) (in Russian).

9. D. Moskal, V. Nadtochy, M. Golodenko, Formation of a periodic structure in GaAs near-surface layer by a laser beam with a diffraction modulated intensity // Functional Materials 13 (2007) (to be published).

10. M. Born, E.W. Wolf, Principles of Optics. Pergamon Press, Oxford, 1991.

11. V. Nadtochy, M. Golodenko, A. Kalimbet, D. Moskal, Structure changes caused by the laser pulse in Ge subsurface layer // Fizika Khimiya Tverd. Tela 4 (3), p. 556-559 (2003) (in Russian).

12. J.F. Nye, Physical Properties of Crystals. Their Representation by Tensors and Matrices. Clarendon Press, Oxford, 1964.

13. J. Hirth, J. Lothe, Theory of Dislocations. McGraw-Hill, New York, 1968.

14. Zhu Le-yi, Huang, Xin-fan, Wang Li, et al., Fabrication of nc-Si array made by pulsed laser constrained interference crystallisation // Chen Kun-ji. Weinadianzijishu=Micronanoelectron. Technol. 39 (7), p. 17-20 (2002). 